\title{
EEllopander
}

(1) Two datasets of 9335 and 3656 probes were run on EXPANDER using their CLICK algorithm. EXPANDER has provided the clustering info which can be seen in 2 and 3 , an expression matrix in 4 and mean cluster pattern in 5 .

(2) Clustering information for 9335 genes provided by EXPANDER.

\section{Clustering Info:}

Algorithm: CLICK

Expected mean homogeneity: $\quad$ Not specified (default) Overall Average Homogeneity: 0.943

ID $\mid$\begin{tabular}{l|l|l|} 
Name & Size & Homogeneity
\end{tabular}

(3) Clustering information for 3565 genes provided by EXPANDER.
(6) Results from clustering 9335 and 3656 probes using EXPANDER's CLICK algorithm. Classification of Oxidative, Reactive Building and Reactive Charging genes.

\begin{tabular}{|c|c|c|c|c|c|}
\hline Clusters (1) & Probes (9335) & OXD (61) & RB (40) & $\mathrm{RC}(45)$ & $\mathrm{NOC}$ \\
\hline 0 & 58 & 0 & 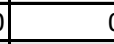 & 1 & \\
\hline Cluster_1 & 9277 & 61 & $4 c$ & 44 & 8 \\
\hline Total & 9277 & 61 & $\overline{4 c}$ & 44 & $14 !$ \\
\hline Missing & $0.62 \%$ & $0.00 \%$ & $0.00 \%$ & $2.22 \%$ & $0.68 \%$ \\
\hline Probe Cluster Ability & & & & & $99.38 \%$ \\
\hline Cluster Collisions & & & & & $57.93 \%$ \\
\hline Sentinal Cluster Efficiency & & $100.00 \%$ & $100.00 \%$ & $100.00 \%$ & $100.00 \%$ \\
\hline Sentinal Clustering Ability & & & & & $99.32 \%$ \\
\hline
\end{tabular}

(4) Expression matrix provided by EXPANDER.

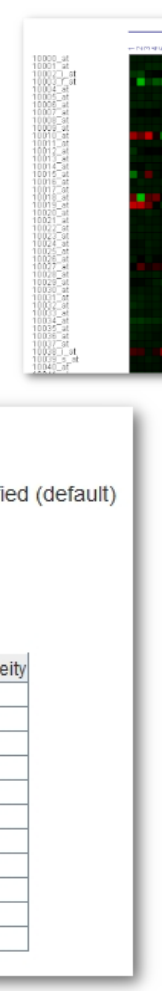

\begin{tabular}{|l|r|r|r|r|r|}
\hline Clusters (11) & Probes (3656) & OXD (55) & RB (40) & RC (41) & NOC \\
\hline Cluster_1 & 1194 & 0 & 0 & 40 & 0 \\
\hline \hline Cluster_2 & 900 & 55 & 0 & 0 & 0 \\
\hline Cluster_3 & 707 & 0 & $\mathbf{4 0}$ & 0 & 0 \\
\hline Cluster_4 & 281 & 0 & 0 & 1 & 0 \\
\hline \hline Cluster_5 & 174 & 0 & 0 & 0 & 0 \\
\hline Cluster_6 & 125 & 0 & 0 & 0 & 0 \\
\hline Cluster_7 & 115 & 0 & 0 & 0 & 0 \\
\hline Cluster_8 & 71 & 0 & 0 & 0 & 0 \\
\hline Cluster_9 & 39 & 0 & 0 & 0 & 0 \\
\hline Cluster_10 & 32 & 0 & 0 & 0 & 0 \\
\hline Cluster_11 & 18 & 0 & 0 & 0 & 0 \\
\hline Total & 3656 & 55 & 40 & 41 & 136 \\
\hline Mission & $0.00 \%$ & $0.00 \%$ & $0.00 \%$ & $0.00 \%$ & 0 \\
\hline Probe Cluster Ability & & & & & $100.00 \%$ \\
\hline Cluster Collisions & & & & & $0.00 \%$ \\
\hline Sentinal Cluster Efficiency & & $100.00 \%$ & $100.00 \%$ & $97.56 \%$ & $99.19 \%$ \\
\hline Sentinal Clustering Ability & & & & & $100.00 \%$ \\
\hline
\end{tabular}

Clustering Info:

Expected mean homogeneity: Not specified (default) Overall Average Homogeneity: 0.76 Overall Average Separation: -0.22 Number of clusters: 11

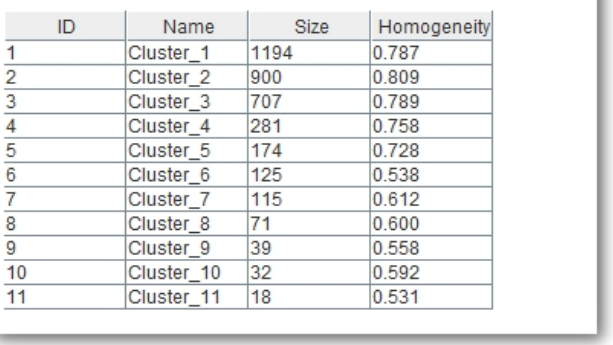

(5) Mean cluster patter for cluster 1 on 9335 probes, provided by EXPANDER.

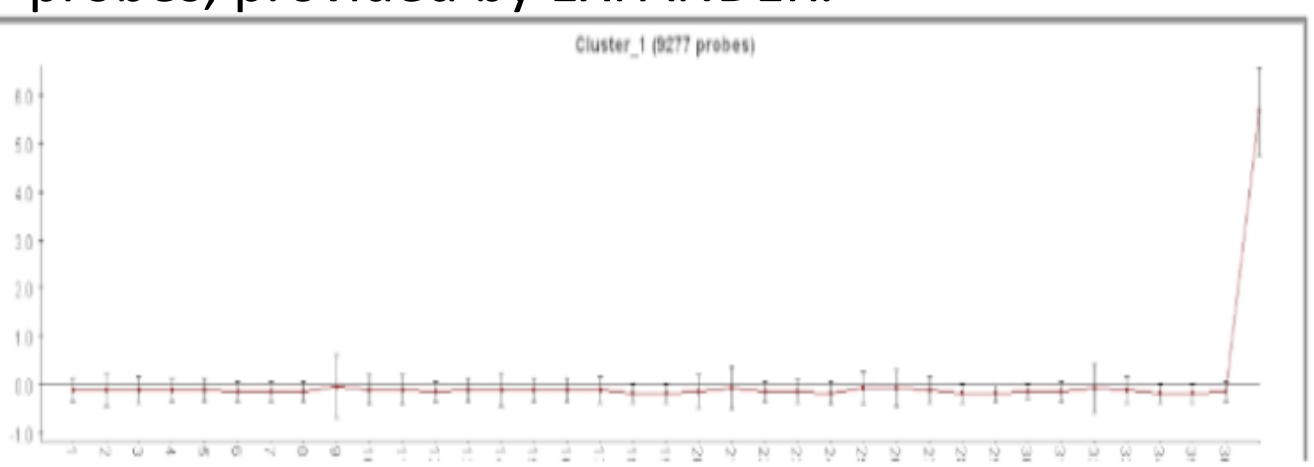




\section{Ellonder}

(1) Two datasets of 9335 and 3565 genes were run on the EXPANDER using their Biclustering algorithm SAMBA. EXPANDER has provided clustering information in 2, clusters for the genes in 3, and expression matrix for each cluster in 4.

(2) Cluster information provided by EXPANDER.

\section{Biclustering info:}

Matrix data: GE Data 21

option files location input: valsp_3ap

Permitted overlap between two biclusters: 0.1

try covering all probes input: false

Take log input: 1

Maximal memory allocated for hashing stage: 100

Maximal kernel size in the hashing stage: 4

Minimal kernel size in the hashing stage: 4

Minimal number of responding probes per condition: 100 Total number of biclusters: 21
(5) Results from clustering 9335 and 3656 probes using EXPANDER's SAMBA algorithm. Classification of Oxidative, Reactive Building and Reactive Charging genes.

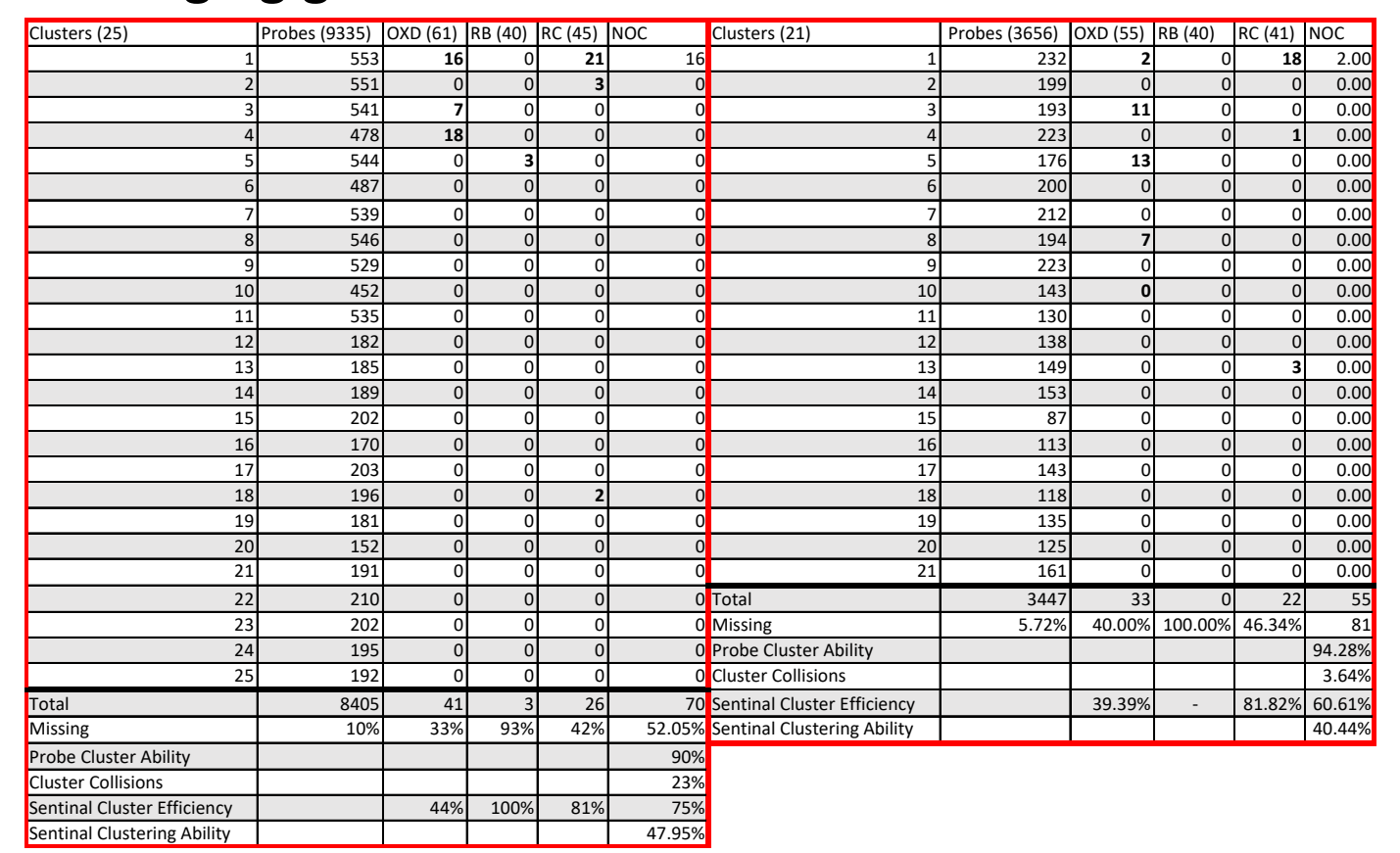

(3) Clustering results from SAMBA provided by EXPANDER.
(4) Expression matrix for Bicluster 15 provided by EXPANDER.

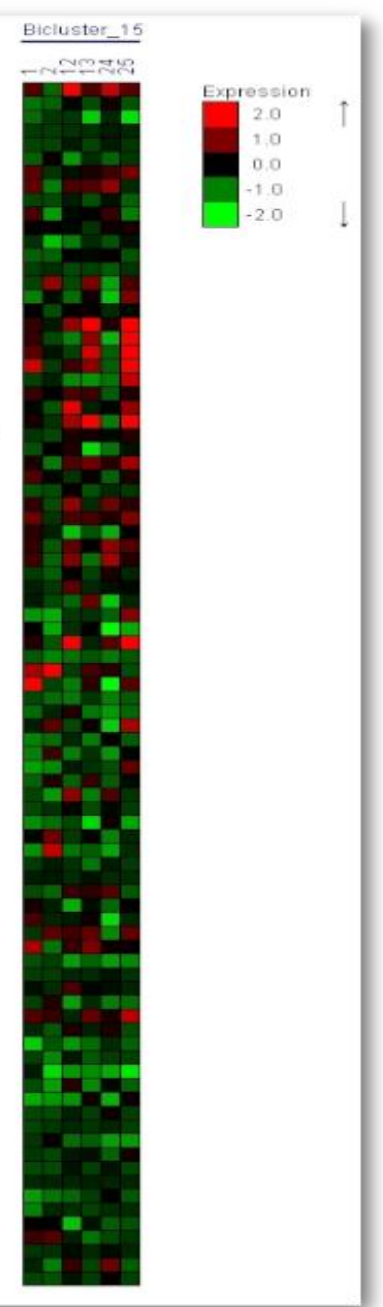




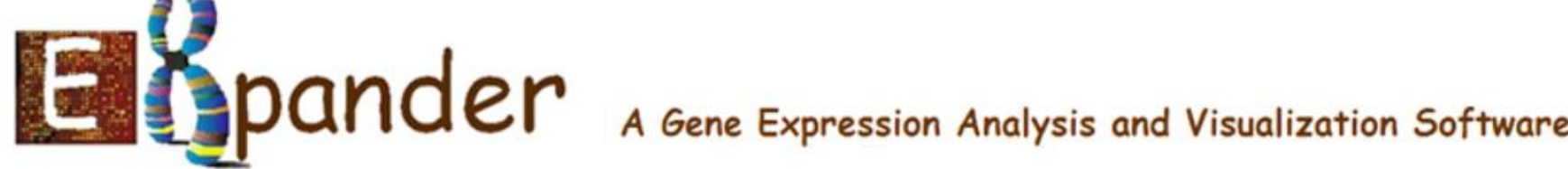

(1) Two datasets of time series gene expression data (of sizes 9335 and 3656) were inputted into EXPANDER and run on the using SOM algorithm. EXPANDER provided cluster information in which can be seen in 2, an Expression Matrix in 3 and mean cluster patterns in 4.

(2) Cluster information provided by EXPANDER.

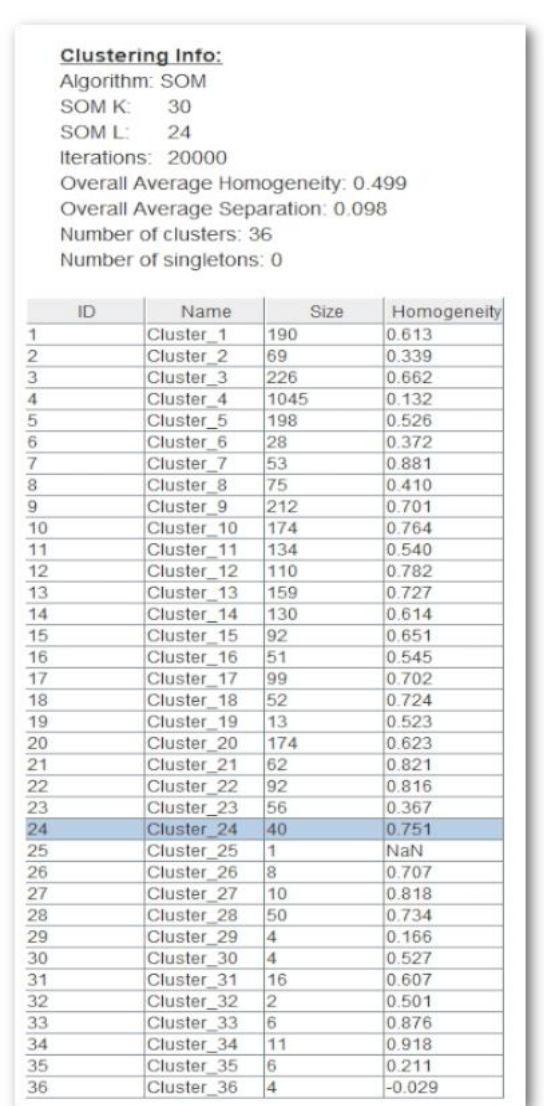

(3) Expression Matrix for cluster 24 provided by EXPANDER.

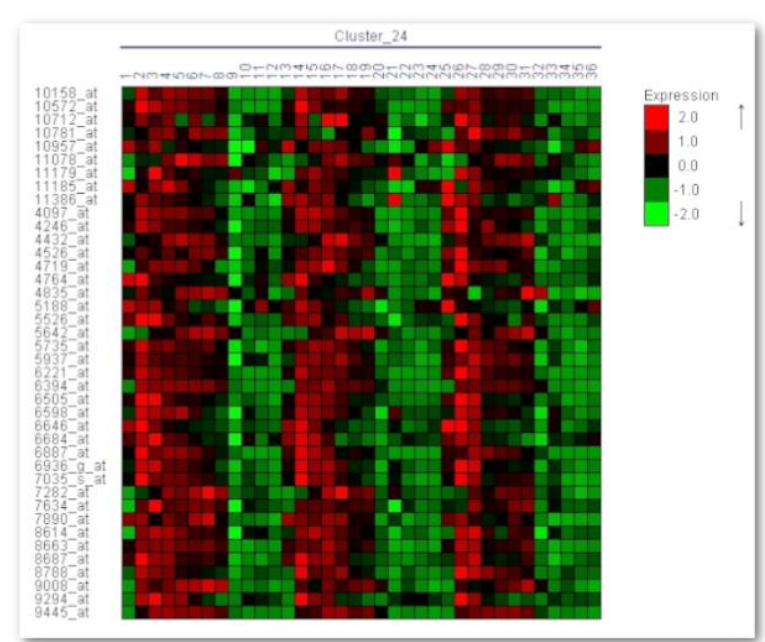

(4) Mean pattern for cluster 24 provided by EXPANDER on the 3656 dataset.

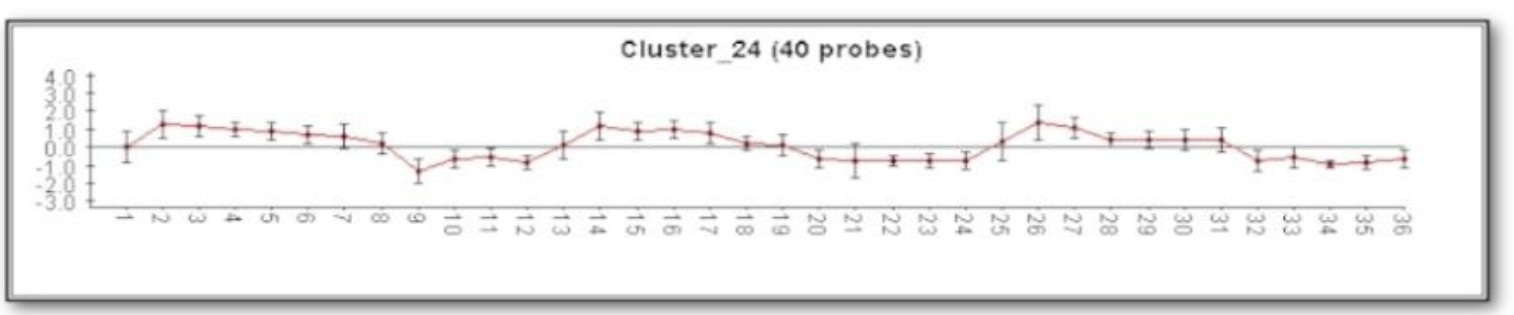

(5) Clustering results from EXPANDER SOM algorithm on 9335 and 3656 probes. Classification of Oxidative, Reactive Building and Reactive Charging genes into

clusters.

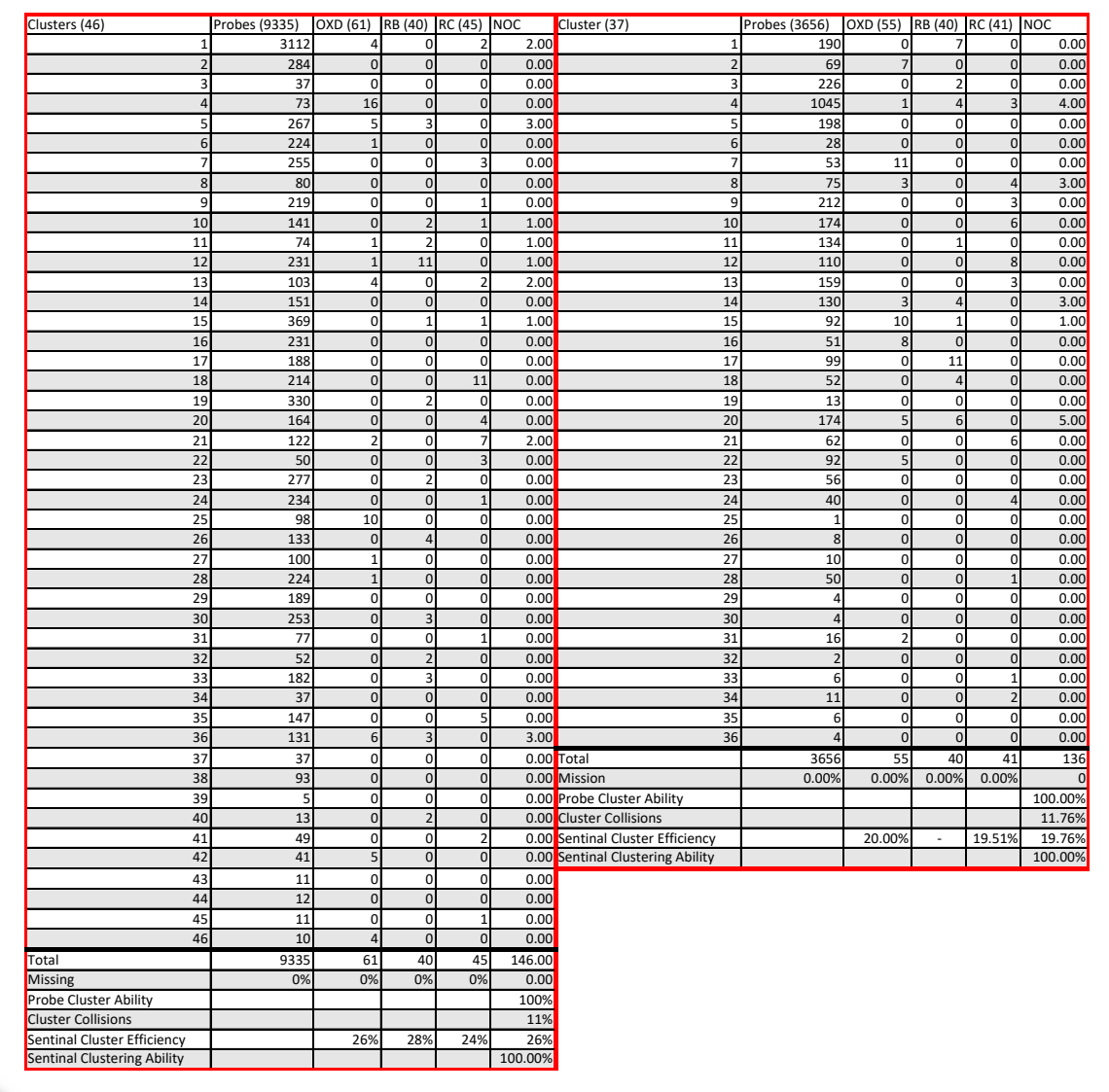

clusters. 
(1) Two datasets of sizes 9335 and 3656 of time-series gene expression data were inputted into the Short Time-series Expression Miner (STEM). The process and results can be seen in $2,3,4$ and 5 .

(2) STEM import window.

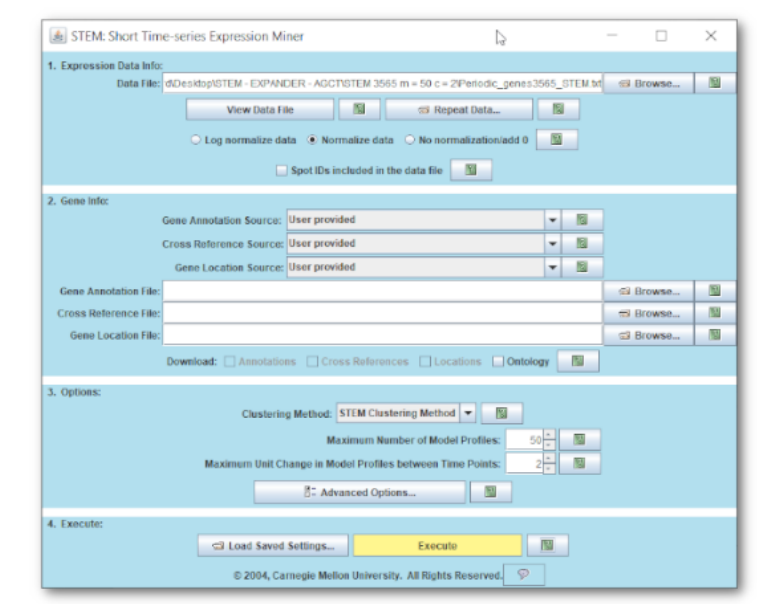

(3) STEM profile window.

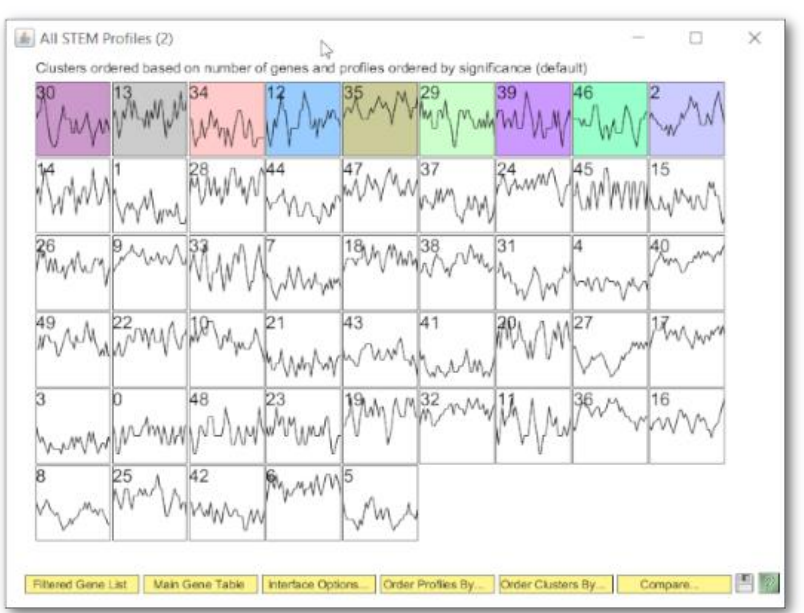

(4) STEM gene expression profile, for profile 30.

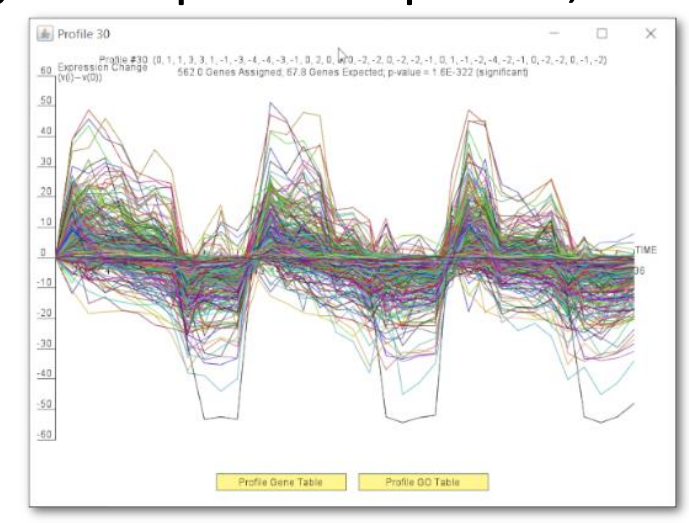

(5) The results of the clustering on the datasets can be seen below. The datasets were run twice once with the ' $\mathrm{m}$ ' parameter set to 3 and 7 , and again set to the default 50 . The table shows number of Oxidative, Reactive and Reactive Charging genes in each cluster.

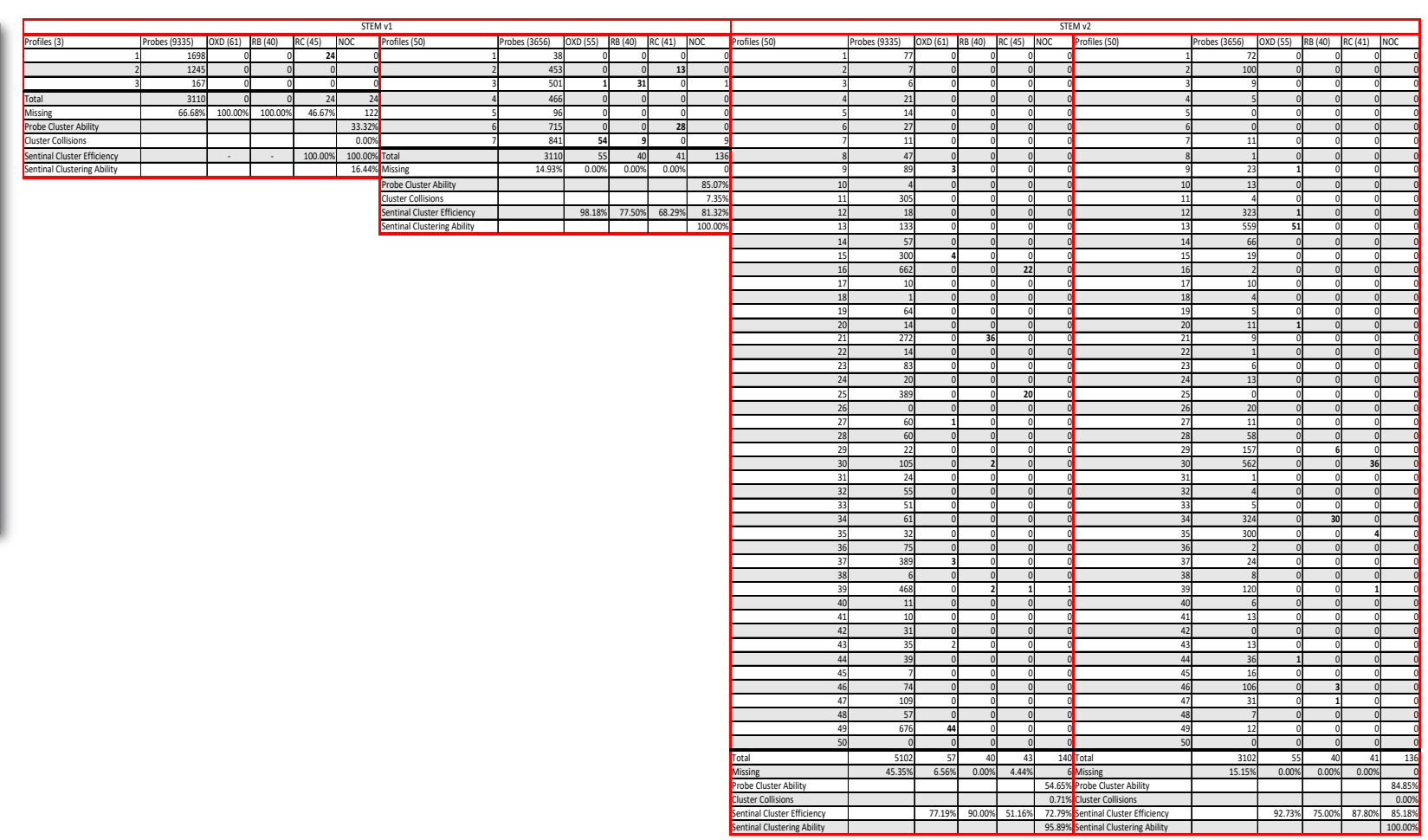


(1) Two datasets of size 3656 probes were inputted into the t-SNE algorithm using the Python Scikit library.

Clustering was done using k-means. The datasets were the original time series gene expression data provided by the probes and the other was the manifold data provided by AGCT from processing the original time series gene expression data. The results can be seen plotted in 2 and 3.
(2) Results plotted from t-SNE on original 3656 probe time series gene expression data.

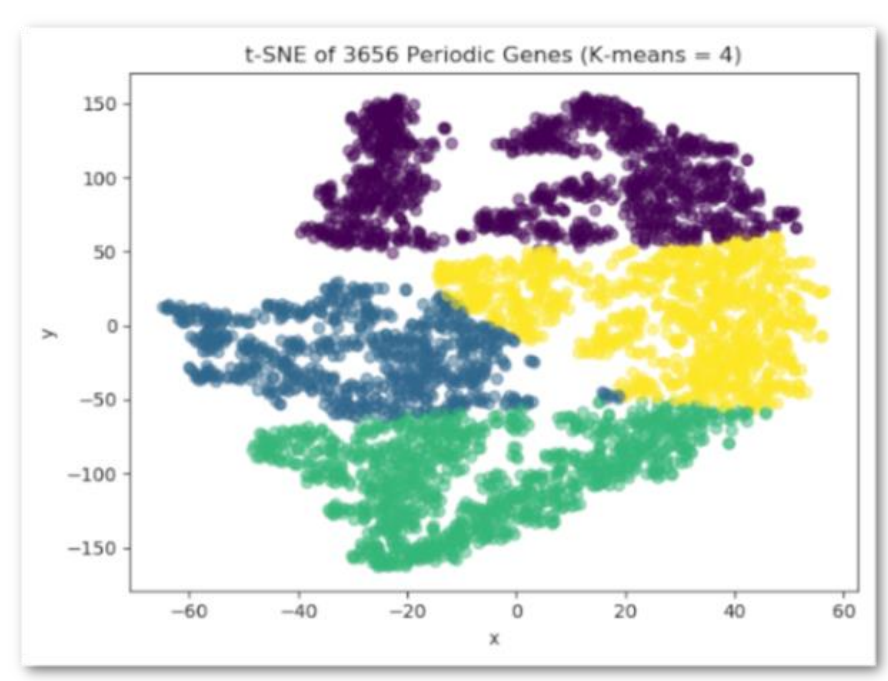

(3) Results plotted from t-SNE using manifold data provided AGCT after using 3656 probe time series gene expression data.

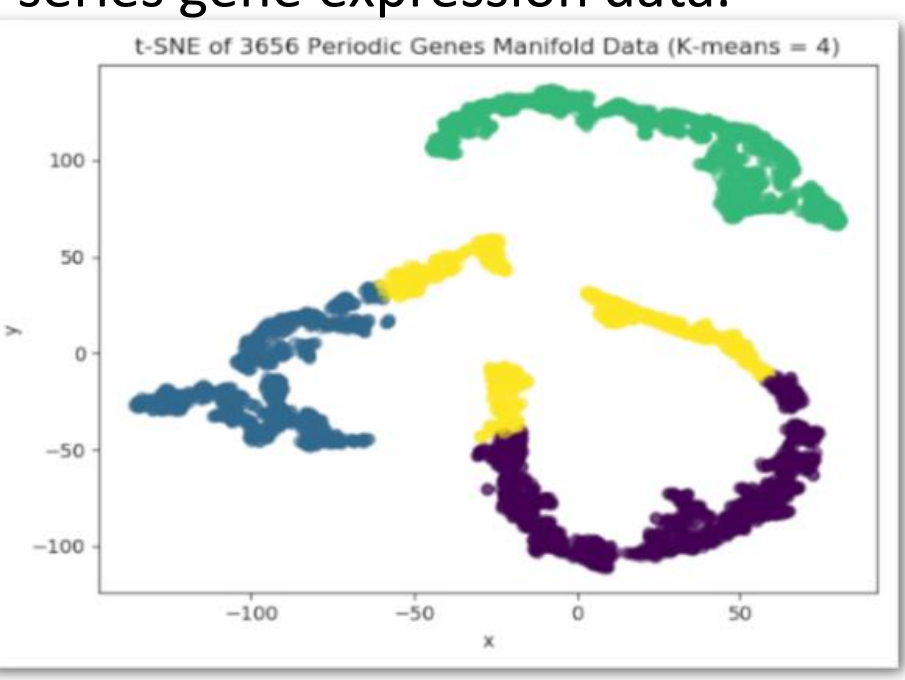

(4) The results of the clustering on the datasets can be seen below. The table shows number of Oxidative, Reactive Building and Reactive Charging genes in each cluster.

\begin{tabular}{|c|c|c|c|c|c|c|c|c|c|c|c|}
\hline \multicolumn{6}{|c|}{ Time Series Data } & \multicolumn{6}{|c|}{ Manifold Data } \\
\hline Clusters (7) & Probes (3656) & OXD (55) & RB (40) & $\mathrm{RC}(41)$ & $\mathrm{NOC}$ & Clusters (7) & \begin{tabular}{|l} 
Probes (3656) \\
\end{tabular} & OXD (55) & RB (40) & $\mathrm{RC}(41)$ & NOC \\
\hline 1 & $\begin{array}{r}407 \\
\end{array}$ & 32 & \begin{tabular}{|l}
0 \\
\end{tabular} & \begin{tabular}{|l|}
18 \\
\end{tabular} & 18 & 1 & $\begin{array}{r}331 \\
\end{array}$ & $\begin{array}{r}0 \\
\end{array}$ & $\begin{array}{r}4 \\
\end{array}$ & 0 & 0 \\
\hline 2 & 569 & 0 & 5 & 5 & 5 & 2 & 683 & 55 & 0 & 0 & $\underline{0}$ \\
\hline 3 & 540 & 0 & 0 & 4 & 0 & 3 & 477 & 0 & 0 & 22 & 0 \\
\hline 4 & 454 & 0 & 1 & 13 & 1 & 4 & 624 & 0 & 0 & 0 & 0 \\
\hline 5 & 640 & 1 & 1 & 1 & 2 & 5 & 425 & 0 & 0 & 6 & $\underline{0}$ \\
\hline 6 & 408 & 17 & 22 & 0 & 17 & 6 & 529 & 0 & 0 & 13 & 0 \\
\hline & 638 & 5 & 11 & 0 & 5 & 7 & 587 & 0 & 36 & 0 & 0 \\
\hline Total & 3656 & 55 & 40 & 41 & 136 & Total & 3656 & 55 & 40 & 41 & 136 \\
\hline Missing & $0.00 \%$ & $0.00 \%$ & $0.00 \%$ & $0.00 \%$ & 0 & Missing & $0.00 \%$ & $0.00 \%$ & $0.00 \%$ & $0.00 \%$ & 0 \\
\hline Probe Cluster Ability & & & & & $100.00 \%$ & Probe Cluster Ability & & & & & $100.00 \%$ \\
\hline Cluster Collisions & & & & & $35.29 \%$ & Cluster Collisions & & & & & $0.00 \%$ \\
\hline Sentinal Cluster Efficiency & & $58.18 \%$ & $55.00 \%$ & $43.90 \%$ & $52.36 \%$ & Sentinal Cluster Efficiency & & $100.00 \%$ & \begin{tabular}{|l|}
$90.00 \%$ \\
\end{tabular} & \begin{tabular}{|l|}
$53.66 \%$ \\
\end{tabular} & $81.22 \%$ \\
\hline Sentinal Clustering Ability & & & & & $100.00 \%$ & Sentinal Clustering Ability & & & & & $100.00 \%$ \\
\hline
\end{tabular}




\section{t-SNE sklearn 9335}

(1) Two datasets of size 9335 probes were inputted into the t-SNE algorithm using the Python Scikit library. The k-means algorithm was used for clustering. The datasets were the original time series gene expression data provided by the probes and the other was the manifold data provided by AGCT from processing the original time series gene expression data. The results can be seen plotted in 2 and 3 .
(2) Results plotted from t-SNE on original 9335 probe time series gene expression data.

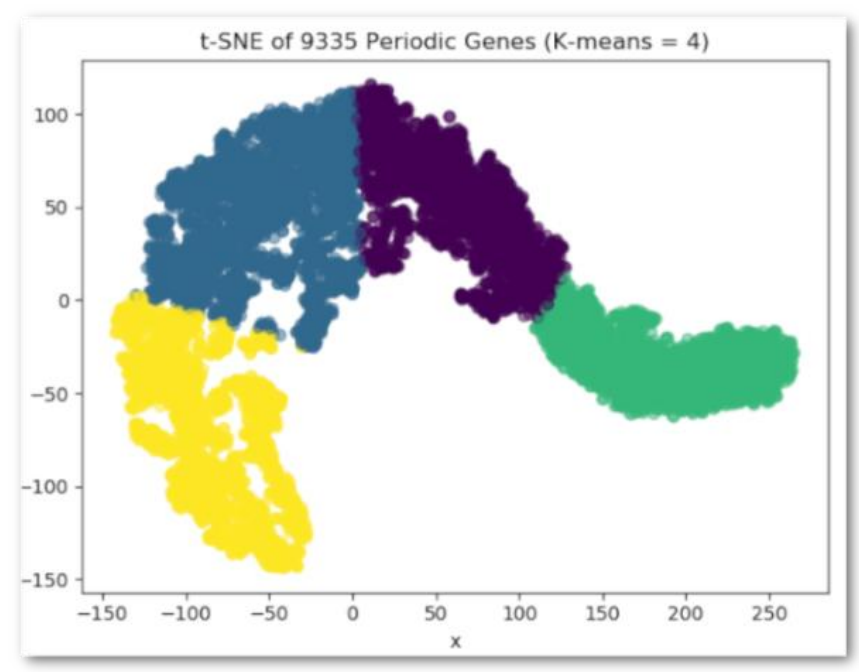

(3) Results plotted from t-SNE using manifold data provided AGCT after using 9335 probe time series gene expression data.

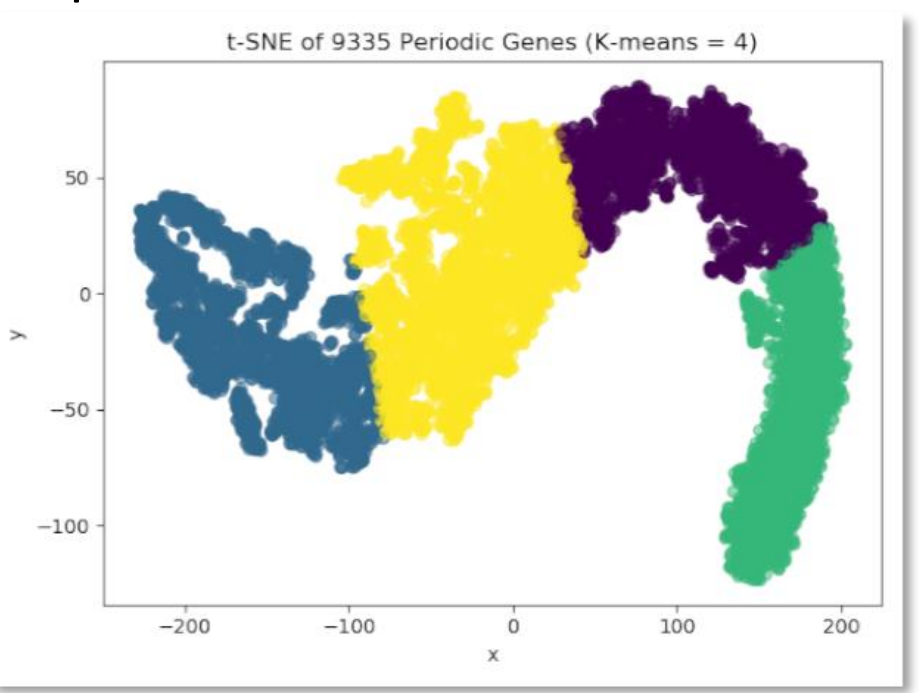

(4) The results of the clustering on the datasets is below. The table shows number of Oxidative, Reactive Building and Reactive Charging genes in each cluster.

\begin{tabular}{|c|c|c|c|c|c|c|c|c|c|c|c|}
\hline \multicolumn{6}{|c|}{ Time Series Data } & \multicolumn{6}{|c|}{ Manifold Data } \\
\hline Clusters (3) & Probes (9335) & OXD (61) & $\mathrm{RB}(40)$ & $\mathrm{RC}(45)$ & NOC & Clusters (3) & Probes (9335) & OXD (61) & RB (40) & $\mathrm{RC}(45)$ & NOC \\
\hline 1 & 3550 & \begin{tabular}{|r|}
3 \\
\end{tabular} & 6 & \begin{tabular}{|r|}
8 \\
\end{tabular} & 9 & 1 & 2657 & 3 & 6 & \begin{tabular}{|r|}
8 \\
\end{tabular} & 9 \\
\hline 2 & 2720 & 4 & 0 & 1 & 1 & 2 & 3140 & 4 & 0 & 1 & 1 \\
\hline 3 & 3065 & 54 & 34 & 36 & 70 & 3 & 3538 & 54 & 34 & 36 & 70 \\
\hline Total & 9335 & 61 & 40 & 45 & 146 & Total & 9335 & 61 & 40 & 45 & 146 \\
\hline Missing & $0.00 \%$ & $0.00 \%$ & $0.00 \%$ & $0.00 \%$ & 0 & Missing & $0.00 \%$ & $0.00 \%$ & $0.00 \%$ & $0.00 \%$ & 0 \\
\hline Probe Cluster Ability & & & & & $100.00 \%$ & Probe Cluster Ability & & & & & $100.00 \%$ \\
\hline Cluster Collisions & & & & & $54.79 \%$ & Cluster Collisions & & & & & $54.79 \%$ \\
\hline Sentinal Clustering Ability & & & & & $100.00 \%$ & Sentinal Clustering Ability & & & & & $100.00 \%$ \\
\hline
\end{tabular}


(1) Two datasets of 3656 probes were inputted into the t-SNE algorithm from Python's Scikit library. The datasets were the original time-series gene expression data, and the manifold data provided by AGCT from processing the original timeseries gene expression data. The results can be seen plotted in 2 and 4 .

(3) Results plotted from Isomap on original 9335 probes time-series gene expression data.

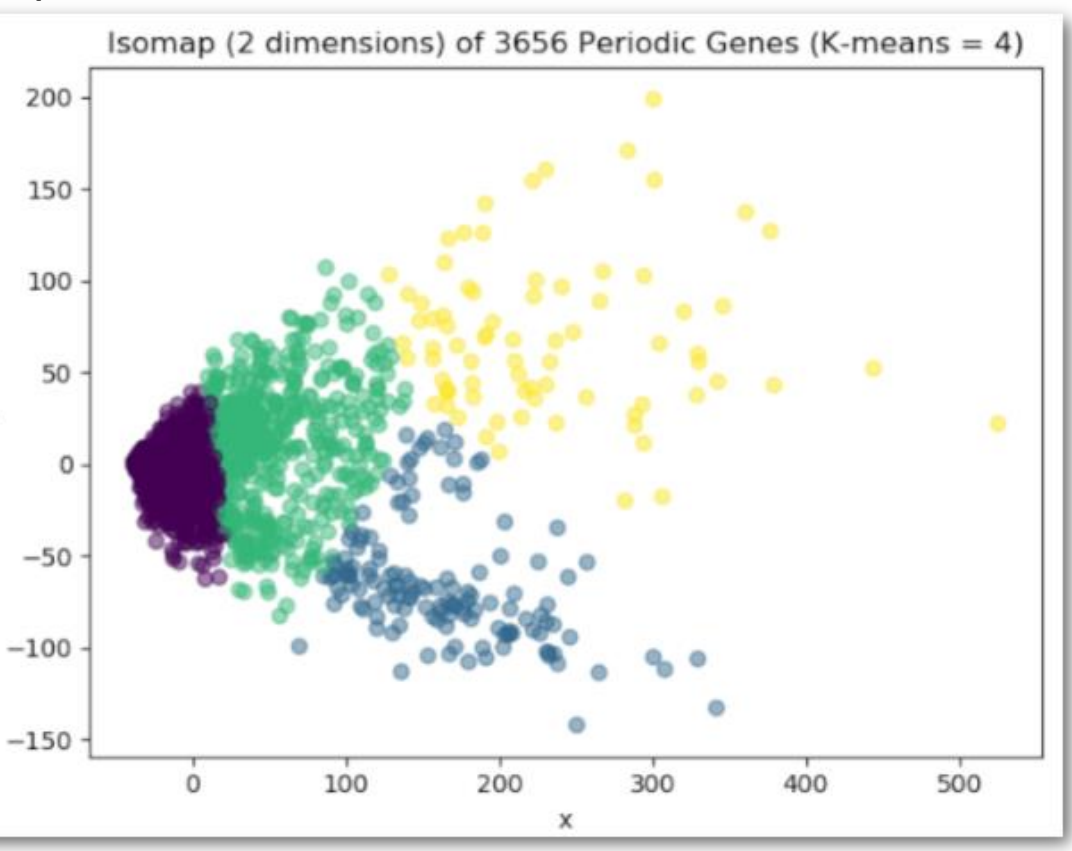

(4) Results plotted from Isomap on original 3656 probes using the manifold data.

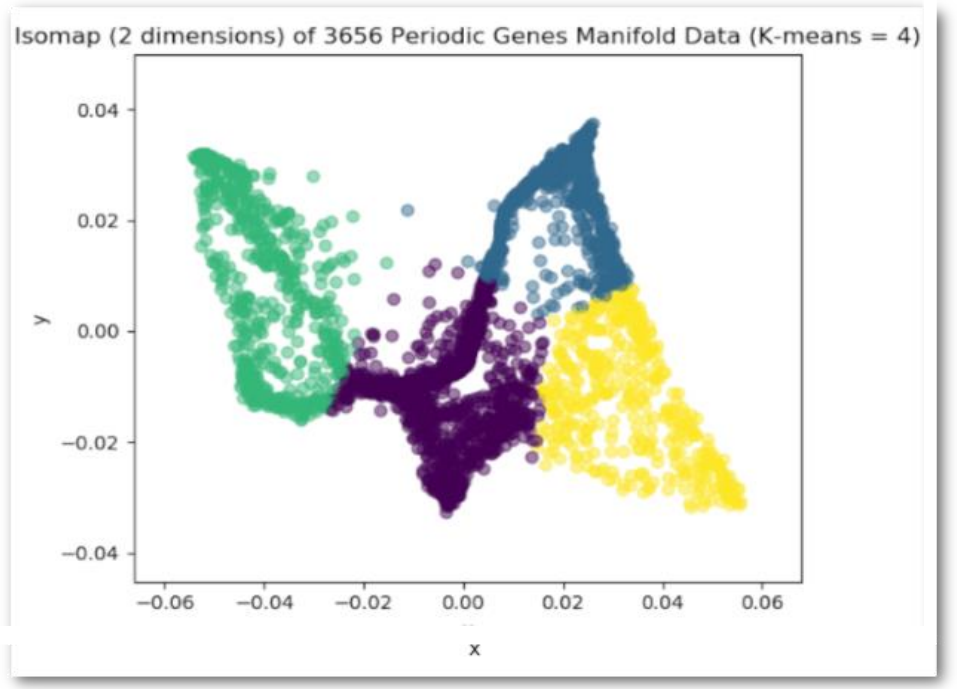

(5) The results from clustering on the dataset can be seen below. The table shows the number of Oxidative, Reactive Building and Reactive Charging genes in each cluster.

\begin{tabular}{|c|c|c|c|c|c|c|c|c|c|c|c|}
\hline \multicolumn{6}{|c|}{ Time Series Data } & \multicolumn{6}{|c|}{ Manifold Data } \\
\hline Clusters (7) & Probes (3656) & OXD (55) & RB (40) & RC (41) & NOC & Clusters (7) & Probes (3656) & OXD (55) & RB (40) & $\mathrm{RC}(41)$ & NOC \\
\hline 1 & 2239 & 5 & 18 & 9 & 14 & 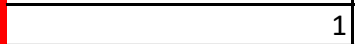 & 297 & 0 & 2 & 5 & 2 \\
\hline 2 & 128 & 0 & 0 & 7 & 0 & 2 & 561 & 8 & 6 & 7 & 13 \\
\hline 3 & 253 & 0 & 0 & 16 & 0 & 3 & 564 & 11 & 7 & 11 & 18 \\
\hline 4 & 79 & 16 & 0 & 0 & 0 & 4 & 335 & 9 & 5 & 2 & 7 \\
\hline 5 & 43 & 0 & 0 & 4 & 0 & 5 & 404 & 5 & 5 & 3 & 8 \\
\hline 6 & 157 & 24 & 0 & 0 & 0 & 6 & 1340 & 20 & 14 & 11 & 25 \\
\hline 7 & 757 & 10 & 22 & 5 & 15 & 7 & 155 & 2 & 1 & 2 & 3 \\
\hline Total & 3656 & 55 & 40 & 41 & 136 & Total & 3656 & 55 & 40 & 41 & 136 \\
\hline Missing & $60.84 \%$ & $0.00 \%$ & $0.00 \%$ & $0.00 \%$ & 0 & Missing & $0.00 \%$ & $0.00 \%$ & $0.00 \%$ & $0.00 \%$ & 0 \\
\hline Probe Cluster Ability & & & & & $100.00 \%$ & Probe Cluster Ability & & & & & $100.00 \%$ \\
\hline Cluster Collisions & & & & & $21.32 \%$ & Cluster Collisions & & & & & $55.88 \%$ \\
\hline Sentinal Cluster Efficiency & & $43.64 \%$ & $55.00 \%$ & $39.02 \%$ & $45.89 \%$ & Sentinal Cluster Efficiency & & $36.36 \%$ & $35.00 \%$ & $26.83 \%$ & $32.73 \%$ \\
\hline Sentinal Clustering Ability & & & & & $93.15 \%$ & Sentinal Clustering Ability & & & & & $100.00 \%$ \\
\hline
\end{tabular}


(1) One dataset of 3656 time-series gene expressions was run using Visgenex. No-Fscoring was used. The process and result can be seen in 2, 3, and 4 . The results of the clustering on the dataset can be seen in 5. The table shows the number of Oxidative, Reactive Building and Reactive Charging genes in each cluster.
(5) Visgenex 2.0 clustering results.

\begin{tabular}{|c|c|c|c|c|c|}
\hline Clusters (4) & Probes (3656) & OXD (55) & RB (40) & $\mathrm{RC}(41)$ & NOC \\
\hline 1 & 925 & 0 & 40 & 0 & 0 \\
\hline 2 & 944 & 0 & 0 & 16 & 0 \\
\hline 3 & 1004 & 55 & 0 & 0 & 0 \\
\hline 4 & 783 & 0 & 0 & 25 & 0 \\
\hline Total & 3656 & 55 & 40 & 41 & 136 \\
\hline Missing & $0.00 \%$ & $0.00 \%$ & $0.00 \%$ & $0.00 \%$ & 0 \\
\hline Probe Cluster Ability & & & & & $100.00 \%$ \\
\hline Cluster Collisions & & & & & $0.00 \%$ \\
\hline Sentinal Cluster Efficiency & & $100.00 \%$ & $100.00 \%$ & $60.98 \%$ & $86.99 \%$ \\
\hline Sentinal Clustering Ability & & & & & $100.00 \%$ \\
\hline
\end{tabular}

(4) Visgenex 2.0 analysis tools.
(2) Visgenex 2.0 gui repository.

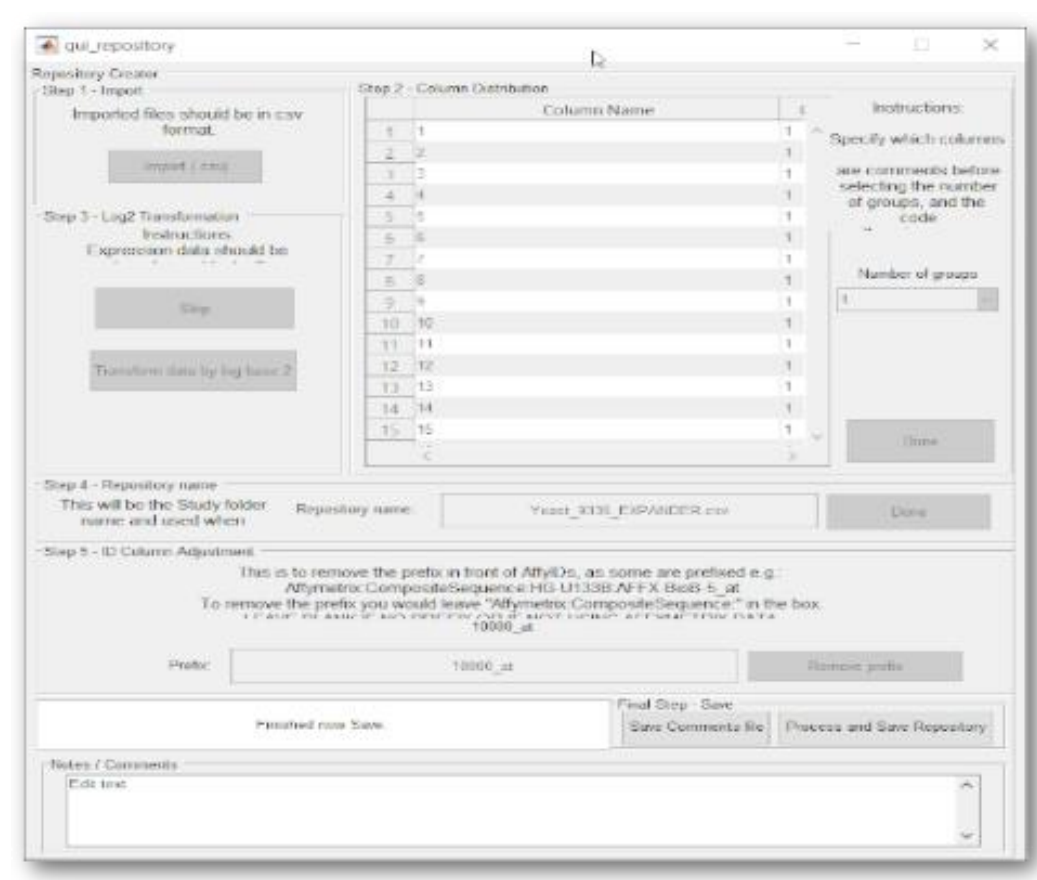

(3) Visgenex 2.0 gui study.

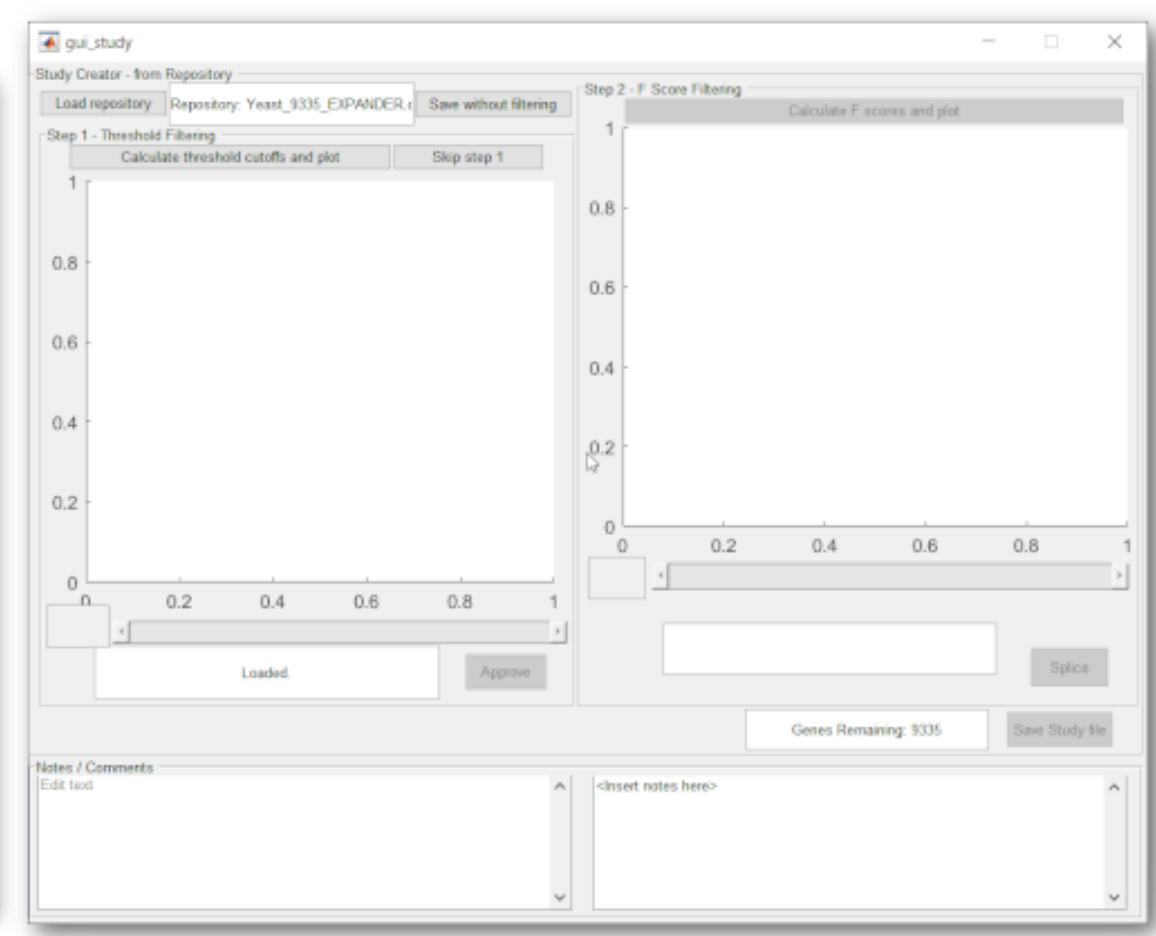

